\title{
Disparate Energy Scaling of Trajectory-Dependent Electronic Excitations for Slow Protons and He Ions
}

\author{
S. Lohmann $\odot^{*}$ and D. Primetzhofer \\ Department of Physics and Astronomy, Uppsala University, Box 516, 75120 Uppsala, Sweden
}

(Received 19 July 2019; accepted 29 January 2020; published 2 March 2020)

\begin{abstract}
We have simultaneously measured angular distributions and electronic energy loss of helium ions and protons directly transmitted through self-supporting, single-crystalline silicon foils. We have compared the energy loss along channeled and random trajectories for incident ion energies between 50 and $200 \mathrm{keV}$. For all studied cases the energy loss in channeling geometry is found lower than in random geometry. In the case of protons, this difference increases with initial ion energy. This behavior can be explained by the increasing contribution of excitations of core electrons, which are more likely to happen at small impact parameters accessible only in random geometry. For helium ions we observe a reverse trend-a decrease of the difference between channeled and random energy loss for increasing ion energy. Because of the inefficiency of core-electron excitations even at small impact parameters at such low energies, another mechanism has to be the cause for the observed difference. We provide evidence that the observation originates from reionization events induced by close collisions of helium ions occurring only along random trajectories.
\end{abstract}

DOI: 10.1103/PhysRevLett.124.096601

Accurate knowledge of the energy deposition by energetic charged particles in matter is imperative for understanding astrophysical phenomena [1,2] and materials modification in extreme environments [3,4]. Knowing the average energy loss per path length provides the basis for analytical techniques such as ion beam analysis, while understanding of subsequent electronically driven processes allows for controlled tailoring of material properties by ion irradiation, implantation, and sputtering, all of which are extensively used in materials research and widely spread in industrial applications [5]. Also, in hadron therapy, the induced energy spectrum of secondary electrons is decisive for a deepened understanding and accurate modeling of nanodosimetry [6].

The common concept for the description of energy dissipation defines the specific energy loss per unit path length as the stopping power $S$ of a material. $S$ is commonly considered a mean value along the trajectory of the ion, and several theoretical concepts have abandoned the complication of an internal structure of the target material and still successfully predicted $S[7,8]$. However, even though we know that the transfer of energy from the moving ion to target constituents is impact parameter dependent [9], the underlying causes, i.e., the exact nature of how energy is

Published by the American Physical Society under the terms of the Creative Commons Attribution 4.0 International license. Further distribution of this work must maintain attribution to the author(s) and the published article's title, journal citation, and DOI. transferred, are insufficiently understood, in particular for slow ions. Advanced theories predicting equilibrium (density functional theory DFT) and nonequilibrium conditions in solids (time-dependent density functional theory TD-DFT) [10] are promising tools to accurately predict energy deposition even at the nanoscale, but still require being benchmarked against suitable experimental data.

Experimental studies of energy loss, however, most often make use of the definition of $S$ and measure an effective average of the specific energy transfers experienced in the individual interactions along a trajectory, often in amorphous or polycrystalline targets. The capability of specifically studying the individual underlying processes in a comparison of experimental data to theory is, therefore, limited. As an example, predictions of energy loss due to binary collisions between the electrons and the ion using DFT typically match the experiment only for protons [11], whereas for heavier ions, due to contributions of more complex, local interactions [12], the observed energy loss is underestimated [13].

By predefining the impact parameters accessed in a specific experiment, a more detailed understanding of electronic excitation channels accessible to an energetic ion can be obtained. A selection of impact parameters can be achieved by employing materials with long-range order, i.e., single-crystalline materials. When an ion enters a crystal with its direction of motion closely aligned with a major crystal axis, it will typically undergo only smallangle collisions with the nuclei. These collisions steer the ion through the channel formed by the strings and planes of 
the crystal, and it moves in an oscillating fashion at large impact parameters [14].

The electronic stopping power of fast light ions in singlecrystalline materials has been studied by several groups [15-21]. At energies between $500 \mathrm{keV}$ and several MeV, significant differences between channeled and nonchanneled trajectories have been reported in silicon targets. In these studies, different experimental approaches like backscattering or transmission with either relatively thick samples or samples with an additional backing layer have been performed. At similar energies, more recent studies with ultrathin, self-supporting silicon foils have focused on the angular distribution of transmitted protons [22-24].

For slower ions with energies in the medium energy regime (several tens to a few $100 \mathrm{keV}$ ) very little systematic analysis exists. Recently, Wang et al. have constructed a transmission channeling setup for a He ion microscope, but no measurements of electronic stopping have been performed [25]. The response from graphene to the impact of slow, highly charged ions has been investigated illustrating the significant influence of charge exchange processes on secondary electron emission in this system [26,27], but no impact parameter dependence of electronic energy deposition has yet been established in such experiments.

At these low energies, the character of ion-solid interaction changes and nonadiabatic processes become important. Thus, in this regime, impact parameter dependent phenomena will behave differently than observed at energies of several hundreds of $\mathrm{keV} /$ nucleon. Specifically, coreelectron excitation is not as efficient, the structure of the density of states of the target becomes important and dynamic processes such as charge exchange [28] and formation of molecular orbitals [29] are expected to significantly contribute and alter the electronic response at the nanoscale, both in terms of number and energy of secondary electrons. Recently, significant advances in theoretical description of these dynamical processes in solids have been made due to the development of TD-DFT. Similar to experiments, also from a theoretical point of view, the order of well-defined lattice locations and the periodicity of crystals make them easier to model than amorphous materials. Under these conditions, TD-DFT has been successfully employed to model transmission of ions through crystalline samples and results on multiple ion-target combinations have been published, for example, $\mathrm{H}$ in $\mathrm{Si}$ and $\mathrm{Ge}$ [30,31], $\mathrm{Si}$ in $\mathrm{Si}$ [32], $\mathrm{Ni}$ in $\mathrm{Ni}$ [33], and $\mathrm{H}$ in graphite [34].

In order to investigate the described dynamics in ionelectron interaction, we simultaneously record angular distributions and energy loss of helium ions and protons transmitted through thin, self-supporting, single-crystalline silicon foils. For the first time we compare helium and proton data at low energies below and around the Bragg peak, respectively. We show that, also for the lowest investigated energies, the energy dissipated along channeled trajectories is significantly less than along random trajectories. As a key result, we find the energy dependence and magnitude of this effect to fundamentally differ between protons and helium ions.

Experiments were performed with the time-of-flight medium energy ion scattering (TOF-MEIS) setup at Uppsala University [35]. Helium ions and protons were detected with a large, position-sensitive microchannel plate detector after transmission through thin, self-supporting Si(100) foils (Norcada Inc. "UberFlat" silicone membranes). Different foil thicknesses were employed to check for possible effects trivially related to trajectory length. The detector was positioned with its surface orthogonal to the initial beam direction, $290 \mathrm{~mm}$ behind the sample. Initial ion energies ranged between 50 and $200 \mathrm{keV}$. The ion energy after transmission through the sample was determined via its flight time simultaneously with a position signal. Rutherford backscattering spectrometry was used to independently confirm thicknesses and purity of the samples.

Figure 1 shows the spatial distribution of ${ }^{4} \mathrm{He}^{+}$ions with initial energies of $200 \mathrm{keV}$ after transmission through a $200 \mathrm{~nm} \mathrm{Si}(100)$ foil. The sample is aligned in such a way that the principal [100] crystal axis is parallel to the incident beam. In this geometry, the vast majority of ions is detected at small scattering angles axially symmetric around the incident beam direction. These ions, thus, travel through the crystal along a channeling trajectory. A minority of ions escape the channels and reach the detector under larger scattering angles (note that the whole diameter of the detector corresponds to deflection angles $\pm 11.5^{\circ}$ ). Some of

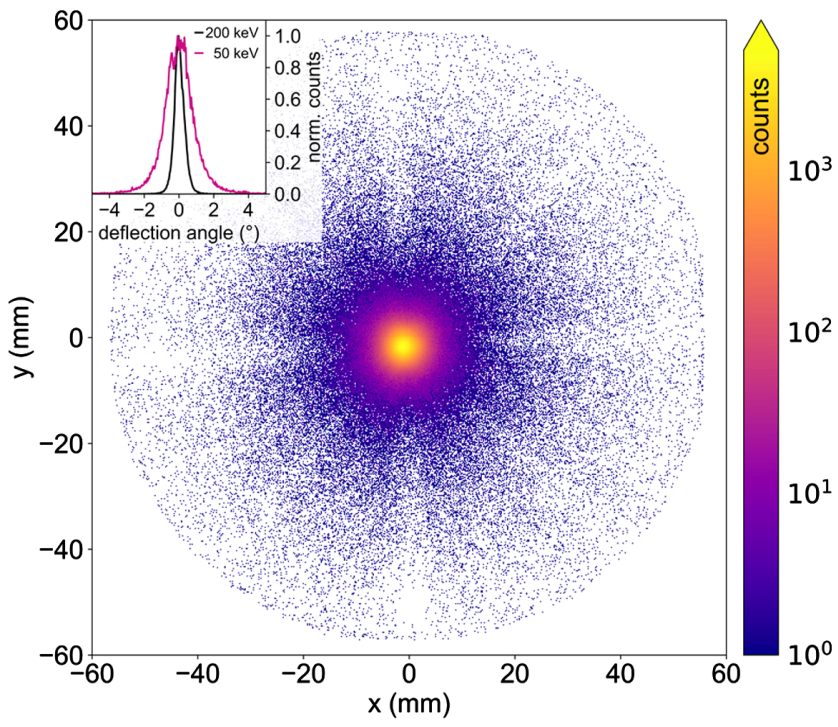

FIG. 1. Spatial distribution of He ions transmitted through a $200 \mathrm{~nm}$ self-supporting $\mathrm{Si}(100)$ foil. The initial ion energy is $200 \mathrm{keV}$ and the center of the detector is positioned at $0^{\circ}$ with respect to the initial beam direction. The main crystal axis is aligned with the beam direction. The inset shows the angular distribution of ions detected along a line parallel to the $x$ axis through the beam center position $(y=-2)$ for initial energies of 200 (black line) and $50 \mathrm{keV}$ (magenta line). 
these trajectories are, however, subject to the blocking effect, which results in reduced intensity at the projections of the crystal axes on the detector. In this way, a real-space image of the crystal structure becomes visible, with information on the particle energy simultaneously available for every pixel.

For Fig. 2 we have turned the sample by $12^{\circ}$ around the $y$ axis and by $7.5^{\circ}$ around the $x$ axis. Otherwise, experimental conditions were identical as for Fig. 1. The beam is not aligned with one of the low-index crystal axes, therefore, we call this geometry "random." Again, the highest intensity can be observed in the center of the detector, i.e., in the direction of the primary beam (denoted by the topmost circle in Fig. 2). The blocking pattern, i.e., the lines and nodes of reduced intensity, is readily visible. Note that the white region on the top is an artifact caused by a cable shadowing the detector. Both graphs also hold an inset showing the angular distribution of the recorded intensity along the $x$ axis. For decreasing energies, the angular spread increases, and the distribution for $50 \mathrm{keV}$ He shown in the inset in Fig. 2 clearly illustrates how initially deflected primary ions can be subsequently channeled. Also, the observed angular spread is found lower for protons as expected (not shown). Generally, distributions of ions of different species and/or initial kinetic energy look qualitatively similar on the detector.

The present data permit us to unambiguously correlate angular deflection and crystal orientation with the associated energy loss distribution. In the following, we assess

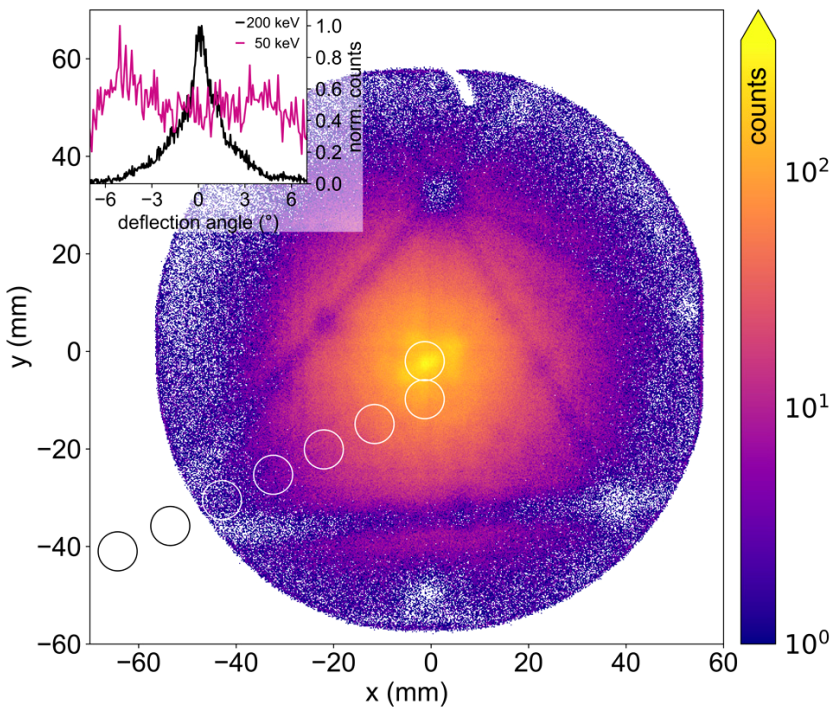

FIG. 2. The sample is turned by $12^{\circ}$ around the $y$ and by $7.5^{\circ}$ around the $x$ axis compared to the channeling alignment used in Fig. 1. Otherwise, experimental conditions were identical. The circles show the position of the incident beams with respect to the crystal axes (visible by the blocking pattern) used for a detailed analysis of the specific energy loss (see text for details). The inset shows again the angular distribution of detected ions for initial projectile energies of 200 and $50 \mathrm{keV}$. the energy loss of ions for different, specific trajectories. For this aim, we varied the beam-crystal alignment successively between the channeled and the random geometry and selected ion trajectories ending in small, circular regions of interest on the detector (radius: $4 \mathrm{~mm}$, scattering angle: $\pm 0.8^{\circ}$ ) around the projected position of the incident beam. We found no difference in energy loss for smaller regions of interest, i.e., for angular deflections small compared to the half-width of the observed channeling phenomenon. The circles in Fig. 2 visualize how the incident beam is positioned with respect to the crystal axes, as made visible by the blocking pattern, in the respective measurement. Note that in practice we rotated the crystalline sample; the detector was not moved, and the evaluated region of interest was kept at a fixed position.

Figure 3 shows the obtained energy loss spectra, i.e., the difference between initial ion energy and final energy, of He ions in $\mathrm{Si}(100)$ for different alignments between beam and crystal axis. In this example, the initial ion energy is $50 \mathrm{keV}$ and the sample thickness is $200 \mathrm{~nm}$. The figure legend shows the degree of crystal rotation away from the (100) channeling geometry $\left(\theta_{x}\right.$ and $\theta_{y}$ denotes the rotation around the $x$ and the $y$ axis, respectively); an effect that is also visualized by the circles drawn into Fig. 2. All curves are normalized to their maximum value to ease comparison.

It is readily visible that the ions lose significantly less energy along channeled than along random trajectories. The broadening of the curves observed in the transition from channeling to random geometry is expected to be

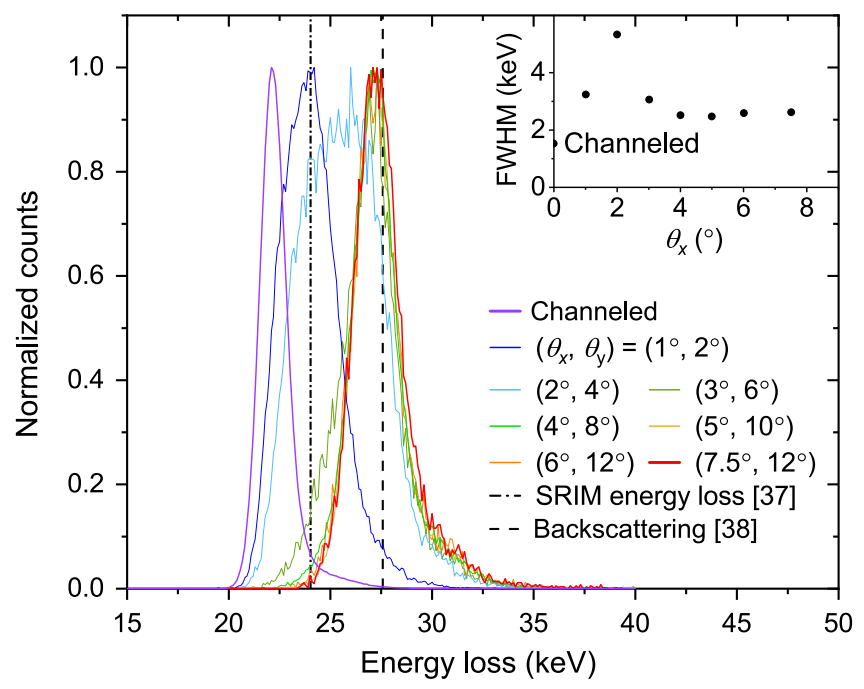

FIG. 3. Energy loss spectra of He ions transmitted through a selfsupporting, $200 \mathrm{~nm}$ thick $\mathrm{Si}(100)$ foil. The initial ion energy is $50 \mathrm{keV}$ and data are taken from the same circular region of interest with $4 \mathrm{~mm}$ radius for each measurement. All curves are normalized to their maximum value. Vertical lines indicate the energy loss in amorphous Si from Refs. [36] and [37]. All other value pairs in the legend give the rotation around the $x$ and the $y$ axis with respect to channeling geometry. The inset shows the observed full width at half maximum of the energy loss distributions. 
caused by an inclusion of qualitatively rather different trajectory types, in analogy to the skimming effect described for backscattering [38].

To quantify this phenomenon and to allow for a comparison between different energies and ion species, we study the energy loss in channeling geometry $\left(\Delta E_{\text {ch }}\right)$ as a fraction of the random energy loss $\left(\Delta E_{r}\right)$. Figure 4 shows the ratio $\Delta E_{\mathrm{ch}} / \Delta E_{r}$ for He ions and protons with initial energies between 50 and $200 \mathrm{keV}$. The error bars take into account the uncertainty of the relative sample-detector distance caused by the finite size of the beam spot and the evaluated region of interest as well as the error in determining the flight time. The latter increases for shorter flight times, i.e., faster ions. The fraction $\Delta E_{\mathrm{ch}} / \Delta E_{r}$ is found smaller than unity for all data points meaning that the energy loss along channeled trajectories is smaller than along random ones in all studied cases. The strength of this effect, however, strongly depends on ion species and initial ion energy. The energy loss of channeled protons at the lowest energies studied reaches about 0.95 of the random energy loss. With increasing energy, the fraction monotonically decreases, i.e., we observe a stronger difference between channeled and random geometries. For helium ions, we see a reverse trend: the channeled energy loss only reaches 0.82 of the random one at $50 \mathrm{keV}$ but increases to about 0.9 at $200 \mathrm{keV}$.

For understanding these results it is necessary to consider the total energy loss as a sum of different processes [40]. The first contribution stems from the energy transferred to

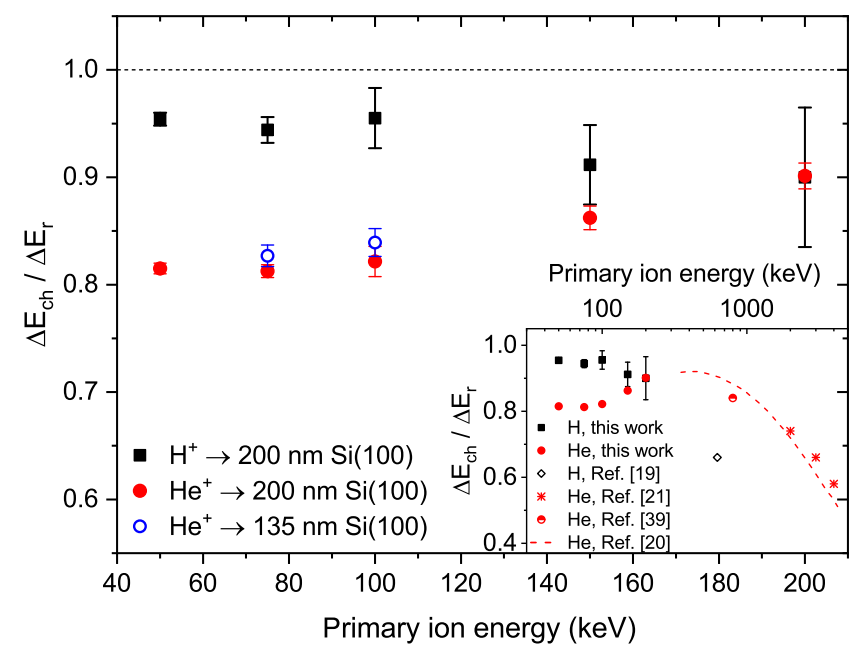

FIG. 4. Energy loss along random $\left(\Delta E_{r}\right)$ and channeled trajectories $\left(\Delta E_{\mathrm{ch}}\right)$ expressed in the fraction $\Delta E_{\mathrm{ch}} / \Delta E_{r}$ for protons (black squares) and for $\mathrm{He}^{+}$ions (red full circles or blue open circles) transmitted through a $\mathrm{Si}(100)$ foil. The inset shows our data in comparison with energy loss ratios measured in $\mathrm{Si}(100)$ from the literature $\left[\mathrm{H}^{+}\right.$data point from Ref. [19] (open diamond), $\mathrm{He}^{+}$data from Ref. [21] (red stars) and Ref. [39] (red half-filled circle)]. The red dashed line corresponds to a guide to the eye through measured data from Ref. [20]. the target valence electrons via binary Coulomb collisions. These electrons can be considered as almost homogeneously distributed inside the target and even within the channel $[18,39,41]$ and, thus, are expected to cause only a marginal difference between random and channeled geometries. Second, at sufficiently high energy transfers in ion-electron collisions, also highly localized silicon core electrons may be excited. Energy loss to these electrons is, therefore, expected to be impact parameter dependent and suppressed for channeled ions, which are steered towards the channel center and experience no close collisions [39]. These effects in combination explain the observed decrease of the ratio $\Delta E_{\mathrm{ch}} / \Delta E_{r}$ in previous studies at high energies and have been extensively discussed in literature [42]. The inset of Fig. 4 shows our results together with datapoints and an interpolation of data discussed in Ref. [20] for $\Delta E_{\mathrm{ch}} / \Delta E_{r}$ at high energies. In this context, the observed energy dependence of our data for protons, as well as its extrapolation to higher energies, can be explained by the increasing efficiency of core-electron excitations and its consequent dominance over valence electron excitations [30].

In contrast, for helium ions with energies below $200 \mathrm{keV}$, the excitation of silicon core electrons becomes even more unlikely than for protons for two reasons. First, the maximum energy transfer in binary electron-ion collisions is much smaller than the binding energies of inner shells. Second, due to the stronger nuclear interaction, typical interaction distances are even larger than for protons also for random geometries. At $200 \mathrm{keV}$ primary energy, scattering by $1^{\circ}$ corresponds, e.g., to an impact parameter of 0.048 and $0.084 \AA$ for protons and He ions, respectively, with expected large impact on the probed core-electron density. Thus, the observed difference in energy loss at these low energies, which is further increasing with lower initial ion energy, i.e., exhibits a maximum between ca. 400 and $500 \mathrm{keV}$, has to be attributed to a different energy loss mechanism.

We propose these observations to be rooted in repeated electron capture and loss of $\mathrm{He}$ projectiles caused by dynamic processes in the atomic level structure not included in a classical free-electron gas framework. Whereas for protons the $1 s$ level is typically resonant with the band in the solid, for He a more complex behavior dependent on interaction time and impact parameters can be expected $[43,44]$. Generally, He ions of initial energies of several tens of $\mathrm{keV} /$ nucleon capture an electron via Auger processes when they enter a solid at any impact parameter [45]. For neutral particles, excited states become relevant for the interaction [46], and a series of ionization processes such as autoionization from an excited state or collision induced reionization, several of which show clear impact parameter dependence, exists. Also, the formation of molecular orbitals and consecutive electron promotion has been shown to be active particularly at lower ion energies as sufficient interaction time is required for these 
nonadiabatic processes to occur [47]. In a global perspective, these processes will introduce a trajectory dependence of the mean charge state at low energies and thus impact electronic stopping.

In the following, we present a qualitative analysis based on these processes allowing us to understand the increase of $\Delta E_{\mathrm{ch}} / \Delta E_{r}$ at low energies based on cyclic capture and loss events affecting directly and indirectly the observed energy loss. The reionization threshold for initially neutral $\mathrm{He}$ projectiles in large-angle collisions from $\mathrm{Si}$ is known from low energy ion scattering experiments [28] and found between 300 and $400 \mathrm{eV}[28,48]$. This energy corresponds to a distance of closest approach of about $0.35 \AA$ for charge exchange during a scattering event, which can only be reached at scattering angles $>0.45^{\circ}$ for $50 \mathrm{keV} \mathrm{He}$ projectiles. From the measured angular distribution of ions (conf. inset Fig. 1) we can deduce that a large fraction of channeled helium could indeed not have undergone charge exchange events, i.e., they are expected to have been neutral for a large part of their trajectory through the target. For a random trajectory the possible number of charge exchange events can be estimated geometrically. The probability to impact a circle with radius $0.35 \AA$ around a silicon nucleus is about 0.04 per monolayer and thus would account to around 40 such close encounters for the whole sample (200 $\mathrm{nm} \approx 1000$ monolayers). In these processes a characteristic energy transfer of about $20 \mathrm{eV}$ is observed [49]. While these direct losses have to be expected, they are clearly insufficient to explain the observed difference in energy loss. However, another consequence of these processes, including autoionization due to formation of molecular orbitals, is that the probability that initially neutral He travels through the target in an ionized state is significantly enhanced for nonchanneling geometries. Assuming a mean Auger neutralization rate similar to that of $\mathrm{Al}$, which is about $5.8 \times 10^{14} \mathrm{~s}^{-1}$ [50], a typical neutralization length of about $23 \AA$ can be expected, effectively introducing a significant difference in the mean charge state for different trajectories. Since the electronic stopping power of the helium ion is significantly higher than that of the neutral atom [51,52], these ionization processes can contribute to the observed differences in the energy loss of ions along channeled and random ion trajectories. On top of that, an energy-dependent charge state ratio will affect energy loss via modification of the interaction potential, as modeled for highly charged ions [53]. For the present system, this process will induce an additional energy-dependent energy loss contribution.

The practical consequences of the described scenario at the nanoscale are that ionization densities of He ions, and other heavier species, are expected to oscillate along typical trajectories in amorphous or polycrystalline solids. This behavior, apart from affecting the observed average energy loss per unit path length, would leave a clear signature in the secondary electron spectrum and subsequent electronically driven cascades. For accurate prediction of such decisive phenomena on the nanoscale, time-dependent modeling has to be improved by including charge exchange and by fully accounting for charge state dependency. Experimentally, measurements of exit charge states are required to obtain a more detailed understanding of these charge exchange mechanisms.

We would like to thank M. Motapothula for providing a $200 \mathrm{~nm} \mathrm{Si}$ sample and for contributing to the very first stages of this project. Accelerator operation is supported by the Swedish Research Council VR-RFI (Contracts No. 821-2012-5144 and No. 2017-00646_9) and the Swedish Foundation for Strategic Research (Contract No. RIF14-0053).

* Corresponding author. svenja.lohmann@physics.uu.se

[1] L. Moroz, G. Baratta, G. Strazzulla, L. Starukhina, E. Dotto, M. A. Barucci, G. Arnold, and E. Distefano, Icarus 170, 214 (2004).

[2] M. E. Palumbo, Astron. Astrophys. 453, 903 (2006).

[3] C. Linsmeier et al., Nucl. Fusion 57, 092007 (2017).

[4] Q. Su, S. Inoue, M. Ishimaru, J. Gigax, T. Wang, H. Ding, M. J. Demkowicz, L. Shao, and M. Nastasi, Sci. Rep. 7, 3900 (2017).

[5] B. Schmidt and K. Wetzig, Ion Beams in Materials Processing and Analysis (Springer-Verlag, Wien, 2013).

[6] M. A. Bernal and J. A. Liendo, Med. Phys. 36, 620 (2009).

[7] J. Lindhard and A. Winther, K. Dan. Vidensk. Selks. Mat. Phys. Medd. 34, 4 (1964).

[8] P. M. Echenique, R. M. Nieminen, and R. H. Ritchie, Solid State Commun. 37, 779 (1981).

[9] W. Brandt, K. W. Jones, and H. W. Kraner, Phys. Rev. Lett. 30, 351 (1973).

[10] E. Runge and E. K. U. Gross, Phys. Rev. Lett. 52, 997 (1984).

[11] J. I. Juaristi, C. Auth, H. Winter, A. Arnau, K. Eder, D. Semrad, F. Aumayr, P. Bauer, and P. M. Echenique, Phys. Rev. Lett. 84, 2124 (2000).

[12] P. Riccardi, M. Ishimoto, P. Barone, and R. A. Baragiola, Surf. Sci. 571, L305 (2004).

[13] M. A. Sortica, V. Paneta, B. Bruckner, S. Lohmann, M. Hans, T. Nyberg, P. Bauer, and D. Primetzhofer, Phys. Rev. A 96, 032703 (2017).

[14] D. S. Gemmell, Rev. Mod. Phys. 46, 129 (1974).

[15] B. R. Appleton, C. Erginsoy, and W. M. Gibson, Phys. Rev. 161, 330 (1967).

[16] F. H. Eisen, G. J. Clark, J. Bøttiger, and J. M. Poate, Radiat. Eff. 13, 93 (1972).

[17] G. Della Mea, A. V. Drigo, S. Lo Russo, P. Mazzoldi, and G. G. Bentini, Phys. Rev. Lett. 27, 1194 (1971).

[18] H. S. Jin and W. M. Gibson, Nucl. Instrum. Methods Phys. Res., Sect. B 13, 76 (1986).

[19] A. Dygo, M. A. Boshart, L. E. Seiberling, and N. M. Kabachnik, Phys. Rev. A 50, 4979 (1994).

[20] J. H. R. dos Santos, P. L. Grande, H. Boudinov, M. Behar, R. Stoll, C. Klatt, and S. Kalbitzer, Nucl. Instrum. Methods Phys. Res., Sect. B 106, 51 (1995). 
[21] R. Mikšová, A. Macková, and P. Malinský, Nucl. Instrum. Methods Phys. Res., Sect. B 406, 179 (2017).

[22] M. Motapothula, Z. Y. Dang, T. Venkatesan, M. B. H. Breese, M. A. Rana, and A. Osman, Phys. Rev. Lett. 108, 195502 (2012).

[23] S. Petrović, N. Nešković, M. Ćosić, M. Motapothula, and M. B. H. Breese, Nucl. Instrum. Methods Phys. Res., Sect. B 360, 23 (2015).

[24] M. Motapothula, S. Petrović, N. Nešković, and M. B. H. Breese, Phys. Rev. B 94, 075415 (2016).

[25] J. Wang, S. H. Y. Huang, C. Herrmann, S. A. Scott, F. Schiettekatte, and K. L. Kavanagh, J. Vac. Sci. Technol. B 36, 021203 (2018).

[26] E. Gruber, R. A. Wilhelm, R. Pétuya, V. Smejkal, R. Kozubek, A. Hierzenberger, B. C. Bayer, I. Aldazabal, A. K. Kazansky, F. Libisch, A. V. Krasheninnikov, M. Schleberger, S. Facsko, A. G. Borisov, A. Arnau, and F. Aumayr, Nat. Commun. 7, 13948 (2016).

[27] J. Schwestka, A. Niggas, S. Creutzburg, R. Kozubek, R. Heller, M. Schleberger, R. A. Wilhelm, and F. Aumayr, J. Phys. Chem. Lett. 10, 4805 (2019).

[28] H. H. Brongersma, M. Draxler, M. de Ridder, and P. Bauer, Surf. Sci. Rep. 62, 63 (2007).

[29] P. Riccardi, A. Sindona, and C. A. Dukes, Phys. Lett. A 381, 1174 (2017).

[30] D. C. Yost, Y. Yao, and Y. Kanai, Phys. Rev. B 96, 115134 (2017).

[31] R. Ullah, F. Corsetti, D. Sánchez-Portal, and E. Artacho, Phys. Rev. B 91, 125203 (2015).

[32] A. Lim, W. M. C. Foulkes, A. P. Horsfield, D. R. Mason, A. Schleife, E. W. Draeger, and A. A. Correa, Phys. Rev. Lett. 116, 043201 (2016).

[33] R. Ullah, E. Artacho, and A. A. Correa, Phys. Rev. Lett. 121, 116401 (2018).

[34] J. Halliday and E. Artacho, Phys. Rev. B 100, 104112 (2019).

[35] M. K. Linnarsson, A. Hallén, J. Åström, D. Primetzhofer, S. Legendre, and G. Possnert, Rev. Sci. Instrum. 83, 095107 (2012).

[36] J. F. Ziegler, M. D. Ziegler, and J. P. Biersack, Nucl. Instrum. Methods Phys. Res., Sect. B 268, 1818 (2010).
[37] T. T. Tran, L. Jablonka, B. Bruckner, S. Rund, D. Roth, M. A. Sortica, P. Bauer, Z. Zhang, and D. Primetzhofer, Phys. Rev. A 100, 032705 (2019).

[38] A. Hentz, G. S. Parkinson, P. D. Quinn, M. A. MuñozMárquez, D. P. Woodruff, P. L. Grande, G. Schiwietz, P. Bailey, and T. C. Q. Noakes, Phys. Rev. Lett. 102, 096103 (2009).

[39] J. H. R. dos Santos, P. L. Grande, M. Behar, H. Boudinov, and G. Schiwietz, Phys. Rev. B 55, 4332 (1997).

[40] P. L. Grande and G. Schiwietz, Nucl. Instruments Methods Phys. Res., Sect. B 136-138, 125 (1998).

[41] M. A. Boshart, A. Dygo, and L. E. Seiberling, Phys. Rev. A 51, 2637 (1995).

[42] C. Cohen and D. Dauvergne, Nucl. Instrum. Methods Phys. Res., Sect. B 225, 40 (2004).

[43] E. C. Goldberg, R. Monreal, F. Flores, H. H. Brongersma, and P. Bauer, Surf. Sci. 440, L875 (1999).

[44] S. Wethekam, H. Winter, D. Valdés, and R. C. Monreal, Phys. Rev. B 79, 195408 (2009).

[45] Y. Kido, T. Nishimura, Y. Hoshino, E. Toyoda, and T. Nakada, Phys. Rev. B 64, 193403 (2001).

[46] F. Matias, R. C. Fadanelli, P. L. Grande, N. E. Koval, R. D. Muiño, A. G. Borisov, N. R. Arista, and G. Schiwietz, J. Phys. B 50, 185201 (2017).

[47] M. Minniti, M. Commisso, A. Sindona, E. Sicilia, A. Bonanno, P. Barone, R. A. Baragiola, and P. Riccardi, Phys. Rev. B 75, 045424 (2007).

[48] R. Souda, M. Aono, C. Oshima, S. Otani, and Y. Ishizawa, Surf. Sci. 179, 199 (1987).

[49] R. Souda and M. Aono, Nucl. Instrum. Methods Phys. Res., Sect. B 15, 114 (1986).

[50] D. Valdés, E. C. Goldberg, J. M. Blanco, and R. C. Monreal, Phys. Rev. B 71, 245417 (2005).

[51] J. I. Juaristi, A. Arnau, P. M. Echenique, C. Auth, and H. Winter, Phys. Rev. Lett. 82, 1048 (1999).

[52] G. Schiwietz and P. L. Grande, Nucl. Instrum. Methods Phys. Res., Sect. B 273, 1 (2012).

[53] R. A. Wilhelm and P. L. Grande, Commun. Phys. 2, 89 (2019). 